PHYSICAL REVIEW D 96, 049906(E) (2017)

\title{
Erratum: Light propagation in the gravitational field of one arbitrarily moving pointlike body in the $2 \mathrm{PN}$ approximation \\ [Phys. Rev. D 94, 124007 (2016)]
}

\author{
Sven Zschocke \\ (Received 30 June 2017; published 10 August 2017)
}

DOI: 10.1103/PhysRevD.96.049906

The geometrical representation in Fig. 1 of the article is incorrect. Therefore, Fig. 1 of the article has to be replaced with the diagram shown here.

We also would like to make an amendment to two sentences of the article.

First, the sentence on page 124007-5 below Eq. (39) should read as follows:

"This fact is related to the case of an arbitrarily moving and radiating electron in classical electrodynamics where the Lienard-Wiechert potentials do not depend on the acceleration of the electron if the worldline of the electron is expressed in terms of its retarded position [50,51]."

Second, the sentence on page 124007-6 below Eq. (45) should read as follows:

"This important fact resembles the case of an arbitrarily moving electron, where the Lienard-Wiechert potentials do not depend on the acceleration as long as the worldline of the electron is given in terms of its retarded position [50,51]."

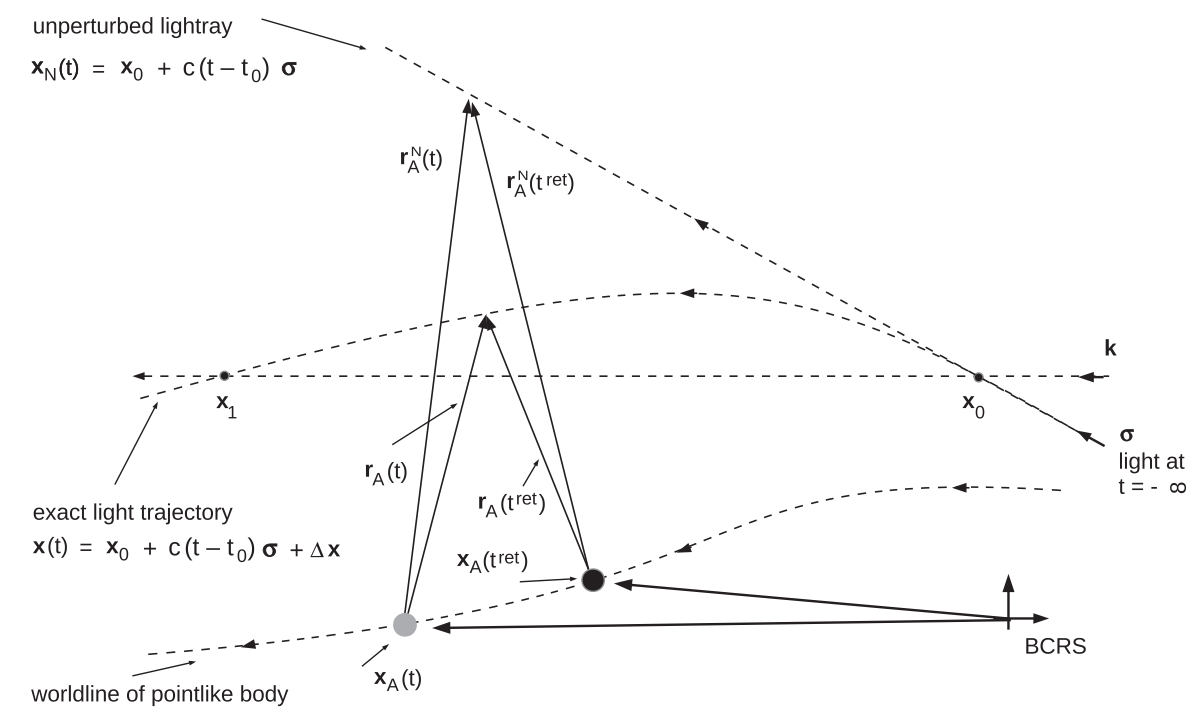

FIG. 1. A geometrical representation of the light trajectory $\boldsymbol{x}(t)$ of Eq. (41) through the gravitational field of one pointlike massive body $A$ moving along an arbitrary worldline in slow motion $v_{A} \ll c$. At the same instant of coordinate time the body's position is $x_{A}(t)$ (gray sphere). However, since gravitational action travels with the finite speed of light, the light ray at $\boldsymbol{x}(t)$ is influenced by the gravitational field generated by the body at its retarded position $\boldsymbol{x}_{A}\left(t^{\text {ret }}\right)$ (black sphere). The spatial vector $\boldsymbol{r}_{A}\left(t^{\mathrm{ret}}\right)$ is defined by Eq. (32) and points from the massive body $A$ at its retarded position toward the exact photon's position at instant $t$. 